\title{
Comparative analisys in cultural test of biological fluids: routine Vs a new strumentation (Alifax HB\&L Uroquattro)
}

Mariapia Galullo', Maria Grazia Castellani', Maria Cacioni', Antonella Repetto²

I Struttura Complessa di Microbiologia, Dipartimento di Medicina Sperimentale e Scienze Biochimiche, Università degli Studi di Perugia, Perugia

2 Struttura Complessa di Microbiologia, A. O. Santa Maria della Misericordia, Perugia

Key words: Biological fluids; Alifax system; Direct cultural analisys

Analisi comparativa dell'esame colturale di liquidi biologici: la routine Vs una nuova strumentazione (Alifax HB\&L Uroquattro)

\section{SUMMARY}

The accuracy and rapidity of a microbiological diagnosis is crucial for the proper management of critical or neutropenic patients. To reduce the time of analysis, we compared the performances of an automated system with those of the conventional method (direct coltural analysis in agar medium BD and a enrichement for aerobic and anaerobic bacteria and fungi in BD bactec bottles). For this study, we evaluated the kind of specimens, the time of analysis and the positivity for several bacterial strains. Finally we compared the specificity and sensitivity of the automated system with those of the traditional coltures. A total of 50 specimens were analysed.All the specimens were from patiens hospitalized in the wards of Perugia's hospital. We found that the results obtained with the Alifax system differed from those of conventional/coltural method. We propose to utilize the Alifax system for coltural analysis of urine where the cut off of significativity is $50 \mathrm{cfu} / \mathrm{mL}$ and the infectious agent involved is often monomicrobic and aerobic. We will continue to use the arrichment of fluid in $\mathrm{BD}$ bottles which is very accurate.

\section{INTRODUZIONE}

L'obiettivo della Microbiologia è quello di fornire al clinico un referto corretto in tempi rapidi.

Solo l'esatta individuazione dell'agente eziologico responsabile di un evento infettivo permette al clinico di somministrare una terapia farmacologica adeguata.

Le condizioni critiche di emergenza di alcune infezioni, portano alla necessità che il referto finale della Batteriologia arrivi in tempi velocissimi al medico, che deve applicare una terapia efficace, personalizzata ed evitare terapie "empiriche" che oggi appaiono pericolose in quanto i batteri sono sempre più resistenti ai chemioterapici.

In questo panorama e con queste finalità, abbiamo approfondito il confronto tra la capacità operativa dello strumento HB\&L Uroquattro della Ditta Alifax, riprogettato ultimamente a livello commerciale, per la finalità dell'esame colturale diretto anche di materiali liquidi, con una metodica routinaria di semina diretta.

Quest'ultima, prevede l'esame colturale in piastre di terreno solido ed arricchimento per batteri aerobi, anaerobi e miceti in flaconi da emocoltura della Ditta Becton Dickinson.

\section{MATERIALI E METODI Campioni biologici}

Lo studio è stato eseguito su materiali liquidi tra $i$ quali: n.10 liquidi cefalo-rachidiani, n.10 liquidi ascitici, n.10 liquidi sinoviali, n.10 liquidi pleurici, n.10 liquidi peritoneali, per un totale di 50 campioni con le due diverse metodiche.

\section{Esame colturale diretto}

L'iter microbiologico di un campione liquido che accede al laboratorio di Batteriologia nella routine, consta di una semina diretta in terreni solidi in piastra quali: Trypticase Soy Agar with 5\% Sheep Blood, Chocolate Agar, Mac Conkey Agar, Columbia CNA Agar with 5\% Sheep Blood, Sabouraud Agar with Chloramphenicol, tutti della ditta Becton Dickinson.

I suddetti terreni, dopo la semina, vengono incubati in termostato a $37^{\circ} \mathrm{C}$ per $18-24$ ore.

$\mathrm{Su}$ ogni campione, si esegue un'osservazione microscopica diretta [esame a fresco $(400 \mathrm{X})$ ] per evidenziare le cellule, leucociti, da correlare all'infezione.

Il materiale viene poi osservato, dopo colorazione di Gram, al microscopio (1000X) per evidenziare

\section{Corresponding author: Antonella Repetto}

Via Orazio Antinori, 21;06123 Perugia

Tel/Fax: 0755784297 - Cell.: 348 77054I9

E-mail: antonella_repetto@libero.it 
la tipologia dei batteri eventualmente presenti.

Un'aliquota dei campioni, laddove è possibile, considerando il quantitativo totale che viene mandato al laboratorio, viene addizionata nelle bottiglie da emocolture per aerobi e miceti della Ditta Becton Dickinson (il volume varia da $1 \mathrm{ml} \mathrm{a} 3 \mathrm{ml}$ ).

Quando il distretto corporeo analizzato può presentare positività anche per batteri anaerobi (ad esempio liquido peritoneale, liquido pleurico ecc...) oltre alla semina in terreni specifici (Schaedler Agar with Vitamin K1 and 5\% Sheep Blood) si esegue l'arricchimento nelle bottiglie per anaerobi sempre della Ditta Becton Dickinson $(1,6,7)$.

Se la bottiglia si positivizza, la sottocoltura viene eseguita sempre in terreno Schaedler ed incubato a $37^{\circ} \mathrm{C}$ per 48 ore dentro una giara con buste per anaerobi (Ditta bioMérieux).

Anche in questo caso i volumi sono gli stessi e sono in stretta relazione alla quantità totale del prelievo inviato al laboratorio.

Se il paziente è già sottoposto a terapia antibiotica, l'arricchimento viene eseguito in bottiglie contenenti resine, in grado di neutralizzare l'effetto dell'antibiotico.

Le bottiglie dell'arricchimento vengono tenute 5 giorni pieni ed incubate nello strumento automatico (FX della Ditta BD)

Nel nostro Laboratorio su tutti i materiali liquidi, in parallelo all'esame colturale, viene eseguita la determinazione del Potere Antibatterico Residuo (P.A.R. Test) su terreno agarizzato addizionato di Bacillus stearothermophilus (Ditta Biolife), tramite dischetti di carta bibula che vengono adesi al terreno e quindi addizionati di $10 \mathrm{uL}$ del liquido da analizzare.

Dopo esecuzione le piastre vengono incubate a $60^{\circ} \mathrm{C}$ per $18-24$ ore.

Infine, per i campioni positivi, viene eseguita, per ogni isolato microbico, l'identificazione e l'antibiogramma tramite strumento Vitek2 della Ditta bioMérieux (Figura I).

\section{Metodica Alifax}

L'apparecchio Alifax è di dimensioni contenute ma ha la possibilità di analizzare fino a 120 campioni contemporaneamente.

Le vials proposte dalla Ditta per l'analisi dei materiali contengono un brodo arricchito che garantisce la crescita anche di microrganismi esigenti ed un'ancoretta che rimescola costantemente il campione, evitando, sedimentazione o galleggiamento dei microrganismi con caratteristiche di adesività particolari (ad esempio Miceti, Staphylococcus spp., ecc...).

La lettura è eseguita tramite un raggio laser che opportunamente polarizzato viene proiettato sul campione.

In tal modo, tramite dei detector che captano il segnale diffuso, si ha traduzione, da parte di un software che tramuta il suddetto segnale, in curve di crescita batteriche.

La macchina ha la possibilità di eseguire anche il P.A.R. Test per ogni campione, in vials specifiche, addizionate con un microrganismo (Staphylococcus spp. coag-negativo) sensibile a tutti i vari antibiotici in commercio.

Il quantitativo di materiale necessario per l'utilizzo dello strumento è di $500 \mu \mathrm{l}$ per l'analisi e altri 500 $\mu l$ per il P.A.R. Test, per un volume totale di $1 \mathrm{ml}$. Infine, la macchina permette all'operatore una personalizzazione che può interessare il profilo di indagine, il protocollo analitico e i tempi di indagine del campione.

La rilevazione della carica batterica tramite tecnica Light Scattering vede rilevazioni di microrganismi con un cut off di $50 \mathrm{cfu} / \mathrm{mL}$.

In relazione alla modalità di lettura che applica l'apparecchio, la tipologia macroscopica dei campioni da analizzare deve avere determinate caratteristiche, infatti si sconsiglia l'utilizzo dell'apparecchio per materiali nettamente ematici o con abbondante cristalluria (Figura II) (2-5).

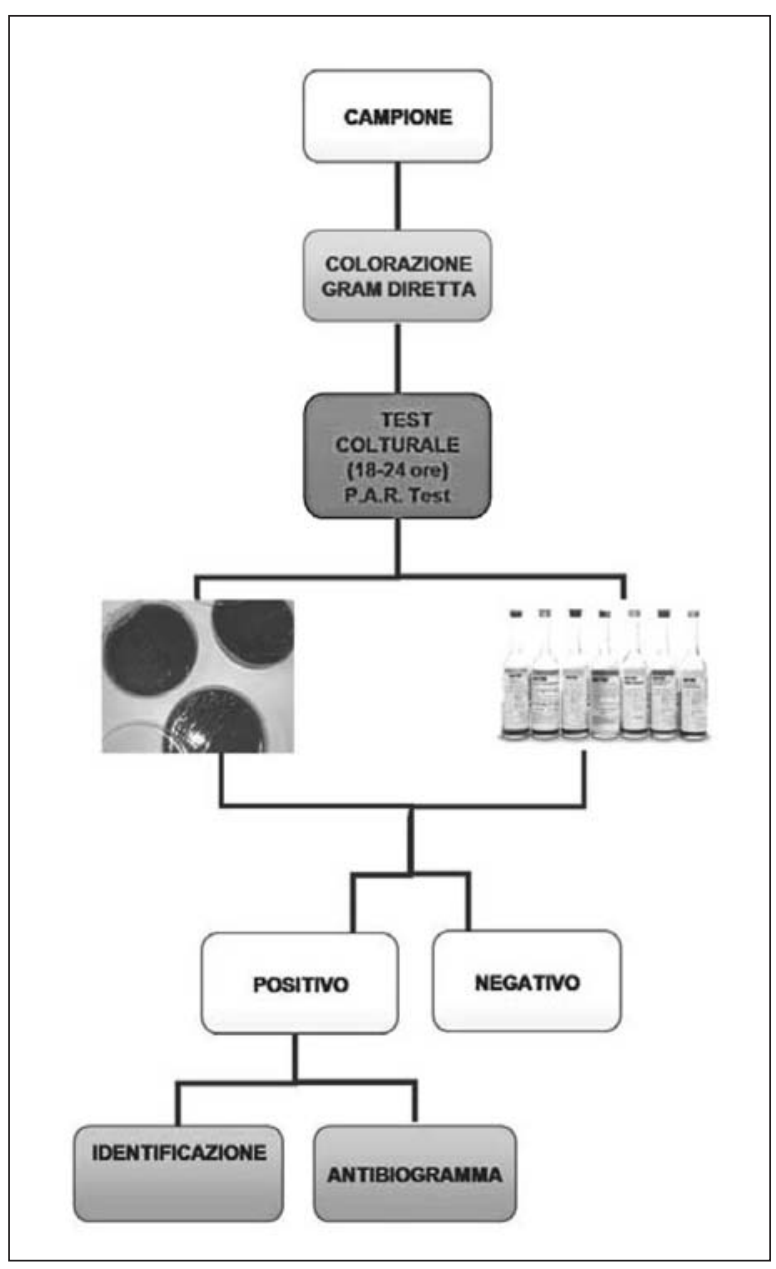

Figura I. Percorso diagnostico dell'esame colturale diretto dei materiali liquidi. 


\section{RISULTATI}

Nella Tabella 1 sono riportati i risultati che mostrano che su 50 campioni il confronto tra le due metodiche ha evidenziato 41 congruenze, ovvero casi in cui l'esame colturale diretto era sovrapponibile all'esame eseguito con l'apparecchio Alifax.

In sei casi la positività rilevata solo con lo strumento era dovuta a batteri probabilmente contaminanti, tutti Staphylococcus spp. coagulasi negativi e nel liquido peritoneale anche un Bacillus spp.. Queste positività sono probabilmente da imputare alle modalità di caricamento del campione e/o alle manipolazioni del materiale prima del caricamento, quindi nella fase pre-analitica e nella fase di accesso al laboratorio.

In due casi si è riscontrata una positività all'esame colturale diretto per anaerobi (un Bacteroides fragilis e un Bacillus subtilis) che la macchina non ha permesso di rilevare, probabilmente per il caricamento del campione le cui modalità avvengono in presenza di ossigeno.

In un caso, positivo all'esame colturale d'arricchimento, la macchina non ha rilevato nessun tipo di crescita, questo probabilmente da attribuire ad una carica batterica troppo bassa. Il campione era un liquor in cui solo l'arricchimento in bottiglia ha rilevato, in terza giornata, la positività per Neisseria meningitidis, altamente patogeno del distretto encefalico considerato, in un paziente già sottoposto a terapia antibiotica, ed in cui anche l'esame colturale diretto era risultato negativo. D'altronde il P.A.R. test positivo, confermava che il batterio era inibito ed in carica troppo bassa.

Soltanto l'arricchimento in bottiglie BD con resine, ha permesso di isolare, identificare ed eseguire l'antibiogramma per l'agente eziologico della meningite in questione.

In nessun campione abbiamo potuto rilevare polimicrobismo e quindi non possiamo trarre conclusioni in merito a questo punto, in quanto il nostro studio ha riguardato tutti materiali positivi per una

Tabella 2. Vantaggi e svantaggi dello strumento Alifax.

\begin{tabular}{|c|c|}
\hline SVANTAGGI & VANTAGGI \\
\hline $\begin{array}{l}\text { NON FORNISCE DISTINZIONE TRA } \\
\text { INFEZIONE POLIMICROBICA E } \\
\text { MONOMICROBICA }\end{array}$ & $\begin{array}{l}\text { TEMPI DI INCUBAZIONE E } \\
\text { SENSIBILITA A PERSONALIZZABILI } \\
\text { DALL'OPERATORE E SECONDO LA } \\
\text { TIPOLOGIA DI CAMPIONE }\end{array}$ \\
\hline $\begin{array}{l}\text { NON PERMETTE LA CRESCITA DI } \\
\text { BATTERI ANAEROBI }\end{array}$ & SEMPLICITẢ DI UTILIZZO \\
\hline INOCULO MINIMO RICHIESTO DI 500 & RAPIDITẢ DI ESECUZIONE \\
\hline $\begin{array}{l}\text { INTERFERENZA DI LETTURA PER } \\
\text { MATERIALI EMATICI O TORBIDI }\end{array}$ & INOCULO STANDARDIZZATO \\
\hline $\begin{array}{l}\text { DIFFICILE DISCRIMINAZIONE TRA } \\
\text { PATOGENI E CONTAMINANTI }\end{array}$ & $\begin{array}{l}\text { VIALS CON TERRENO ARRICCHITO } \\
\text { CON MIXING CONTINUO }\end{array}$ \\
\hline $\begin{array}{c}\text { DOPO L'ANALISI CON LO } \\
\text { STRUMENTO VA ESEGUITO L'ESAME } \\
\text { COLTURALE PER L'IDENTIFICAZIONE } \\
\text { E L'ANTIBIOGRAMMA }\end{array}$ & RISULTATI QUALITATIVI \\
\hline & P.A.R. TEST AFFIDABILE \\
\hline
\end{tabular}

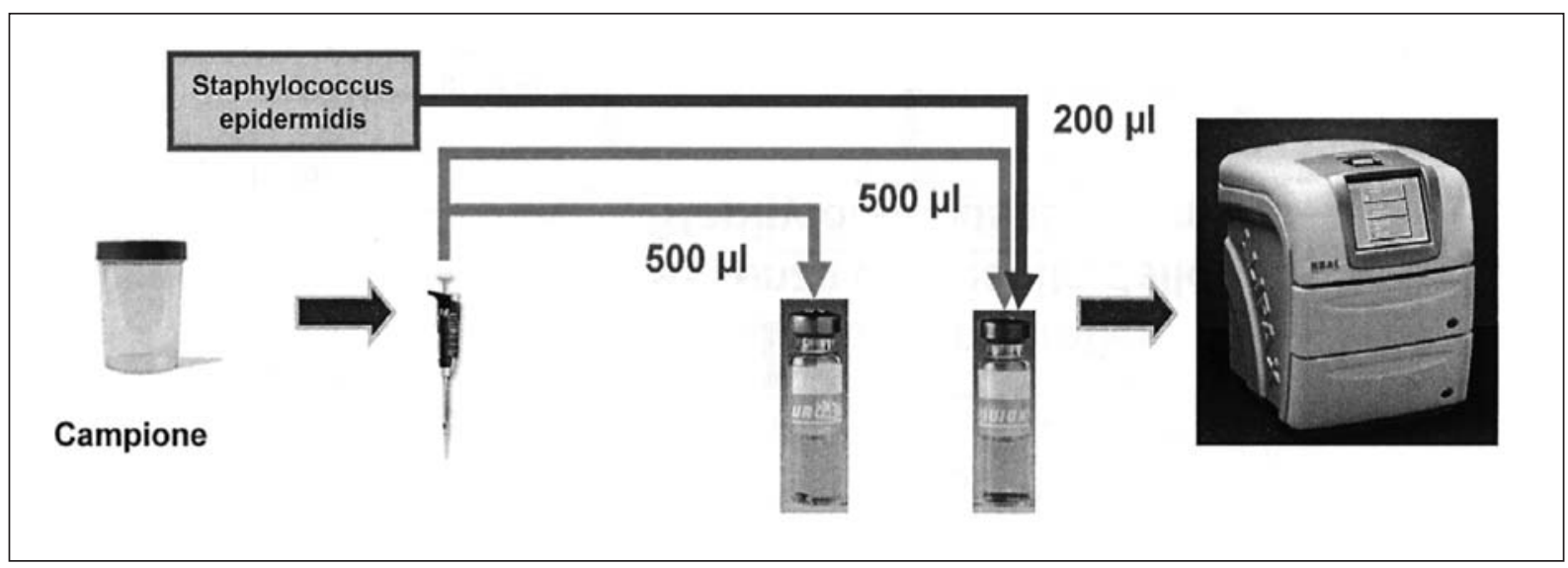

Figura II. Percorso diagnostico di liquidi biologici con strumento Alifax.

Tabella I. Congruenze e microrganismi riscontrati dal confronto dell'esame colturale diretto con lo strumento Alifax

\begin{tabular}{lccc}
\hline Materiali & Positivi solo con Diretto & Positivi solo Alifax (contaminazioni) & Congruenze \\
\hline n. I 0 Liquor & I N. meingitidis & I Staphylococcus spp coag. Neg. & 8 \\
\hline n. I0 L. Pleurico & I Staphylococcus spp coag. Neg & 9 \\
\hline n. I O L. Peritoneale & $\begin{array}{c}\text { I Staphylococcus spp coag. Neg } \\
\text { I Bacillus spp. }\end{array}$ & 8 \\
\hline n. I 0 L. Ascitico & $\begin{array}{c}\text { I Bacteroides fragilis } \\
\text { I Bacillus subtilis }\end{array}$ & I Staphylococcus spp coag. Neg & 7 \\
\hline n. I O L. Sinoviale & & I Staphylococcus spp coag. Neg & 9 \\
\hline
\end{tabular}


singola specie microbica, ma ricordiamo che l'analisi con la macchina è di tipo qualitativo.

Per quanto riguarda la lettura dei campioni con, la personalizzazione possibile dello strumento Alifax, la nostra valutazione è stata eseguita sia 6 che 18 ore. I risultati non hanno portato a differenze allungando i tempi di lettura, ciò è di indubbio interesse per dare degli orientamenti sulla positività del campione in tempi rapidi.

Anche la determinazione del P.A.R. test eseguito con la metodica diretta e con l'apparecchio non ha dato nessuna incongruenza al confronto dei due metodi.

\section{CONCLUSIONI}

Riassumendo, nella Tabella 2 sono riportati i vantaggi e gli svantaggi dello strumento della Ditta Alifax.

Appare evidente come l'apparecchio è sicuramente duttile, semplice ma può dare dei falsi positivi in relazione al caricamento.

Secondo noi, in ogni modo può dare una risposta orientativa al clinico, soprattutto nelle situazioni più difficili e quando è sicuramente importante iniziare una terapia antibiotica precoce.

Gli svantaggi portano a dire che, secondo noi, il campione va in ogni modo valutato con un esame colturale diretto per evidenziare polimicrobismo, batteri anaerobi e possibili contaminazioni.

La Ditta negli ultimi mesi propone lo strumento con la possibilità di eseguire anche un antibiogramma di pronto intervento sui materiali positivi; in realtà propone questa metodica per campioni positivi dal flacone dell'emocoltura. Casi questi in cui la positività è rilevata già da strumenti automatici ed è spesso monomicrobica.
Il cut off di applicazione di $50 \mathrm{cfu} / \mathrm{ml}$ proposto dalla Ditta non ci convince per materiali delicati ed in pazienti sottoposti a terapie antibiotiche massicce come per esempio pazienti neutropenici. Concludendo, riteniamo che la macchina possa essere applicata con vero successo nell'analisi dell'urina, infatti in questo liquido biologico il cut off proposto è sicuramente buono anche in quelle situazioni dove l'eziologia della cistite si studia in pazienti con catetere urinario, neutropenici o già in terapia antibiotica.

\section{BIBLIOGRAFIA}

1. Bourbeau P, Riley J, Heiter BJ, Master R, Young C, Pierson C. Use of BacT/Alert blood culture system for culture of sterile body fluids other than blood. J Clin Microbiol 1998; 36: 3273-7.

2. Branca G, Plaisant P, Archibusacci C, Franco A, Fadda G. Application and Exsperiences with the UROQUICK instrument in a Microbiology Laboratory from October 1998 to August 2000, Poster, SIM, Jesi 2000.

3. Camporese A. Il Microbiologo clinico nel panorama di modernizzazione della medicina di laboratorio. 2004.

4. Di Taranto A, Mosca A, Carucci A, Antonietti R, Miragliotta G. Valutazione dello strumento automatico URO-QUICK per lo studio della Batteriuria, Poster, 31st AMCLI, Rimini 2002, Microb Med. 2002; 17.

5. Fontana $\mathrm{C}$, et al. Coltura ed arricchimento dei campioni biologici liquidi: utilizzo dell'Uro-Quick (Alifax), Poster, National Congress SIM, Milano 2004.

6. Runyon BA, Umland ET, Merlin T. Inoculation of blood culture bottles with ascitic fluid. Arch Intern Med 1987; 14: 73-5.

7. Singh N, Rihs JD, Gayowski T, Mieles L, Yu VL. Improved detection of spontaneous bacterial peritonitis with Bactec as compared with conventional culture methods: A prospective study. Diagn Microbiol Infect Dis 1994; 19: 1-4. 